\title{
Strategy for postmortem diagnosis of myocardial infarction
}

\author{
Atsushi Kurata ${ }^{1}$ (1D \\ Published online: 14 September 2019 \\ (C) Springer-Verlag GmbH Germany, part of Springer Nature 2019
}

In this issue of Virchows Archiv, Michaud and colleagues, on behalf of the Association for European Cardiovascular Pathology (AECVP), provided a timely and comprehensive review article on postmortem diagnosis of myocardial infarction (MI) [1]. They not only compiled classical histological features, but also reflected very recently updated clinical classification. Furthermore, recent developments in postmortem imaging and biomarkers including immunohistochemistry in MI pathology are presented.

Based on this review, practical diagnostic workflow can be conceivable from the standpoint of forensic pathologists (Fig. 1). This workflow includes cases of sudden cardiac death, in which coronary atherosclerotic disease is a leading cause even in young people ( $\leq 35$ years) $[2,3]$. Postmortem diagnosis of MI requires careful differentiation, using comprehensive clinicopathological approach. First, detailed clinical information including follow-up data (e.g., cardiac troponin levels, ECG changes) should be obtained. Risk factors of MI (e.g., hyperlipidemia, diabetes mellitus) can be known even in cases with sudden death not followed in the hospital. Second, evidence of death from heart failure in other organs should be collected at autopsy such as lung congestion and hypoxic hepatic necrosis.

Necrosis of cardiomyocytes is a crucial finding at MI autopsy, but earliest findings of myocardial injury including contraction band necrosis may be subtle and/or nonspecific before evident neutrophilic infiltration occurring 6 to $12 \mathrm{~h}$ after the onset of the ischemic attack [4]. Immunohistochemical markers may serve as early detection, but it takes at least $1 \mathrm{~h}$ after the onset of the attack [4]. Next, if the extent of the myocardial necrosis matches

See related article https://doi.org/10.1007/s00428-019-02662-1

Atsushi Kurata

akurata@tokyo-med.ac.jp

1 Department of Molecular Pathology, Tokyo Medical University, 6-1-1, Shinjuku, Shinjuku-ku, Tokyo 160-8402, Japan subendocardial region or perfusion territory of the coronary arteries, MI can be successfully diagnosed, but otherwise other diseases including myocarditis would be taken into consideration. On such occasions, searching for the type and distribution of infiltrating inflammatory cells may be helpful [5]. If there is an old MI, the possibility that it caused a fatal arrhythmia must also be considered [6]. On the other hand, one study revealed that crosssectional examination across at 3-mm intervals of coronary arteries in 33 cases with old MI detected plaque rupture in 18 cases $(55 \%)$ [7].

Therefore, serial examination of coronary arteries is advisable in order not only to confirm the cause of MI already evidenced by myocardial necrosis but also to explore possibility of MI more deeply in cases with no myocardial necrosis. It has long been accepted that critical narrowing $\geq 75 \%$ of major epicardial coronary arteries indicates culprit of ischemic heart disease $[3,6]$, but this critical narrowing may be asymptomatic [6]. In such cases, reevaluation of the myocardium is advisable. Contraction band necrosis, albeit nonspecific as mentioned above, may be helpful to strengthen the possibility of MI if this feature is found consistent with perfusion territory of the narrowed coronary artery [2].

If neither myocardial necrosis nor critical coronary stenosis is observed, other causes that brought about MI are to be considered. Coronary lesions do not always act alone to cause ischemic injury, but can lead to it in relation to other findings. For example, significant hypertrophy of the heart with even a moderate degree of coronary stenosis may be sufficient to cause a fatal arrhythmia [2]. Alternatively, post-mortem coronary angiography may discover collaterals that weaken the influence of coronary stenosis. Therefore, comprehensive clinicopathological judgement is required. Identification of rupture-prone vulnerable plaque in the coronary arteries, which is composed of large lipid core and thin fibrous cap [8], may spur forensic pathologists to further investigate cross-sectional coronary examination. It was reported that one of the characteristics of vulnerable plaque is immaturity of smooth muscle cells 
Fig. 1 Diagnostic workflow at autopsy in cases suspected to be myocardial infarction (MI).

Tables and figures correspond to those presented in the article by Michaud and colleagues [1]. Abbreviations: c/w, compatible with; $\mathrm{D} / \mathrm{D}$, differential diagnosis; s/o, suspicious of

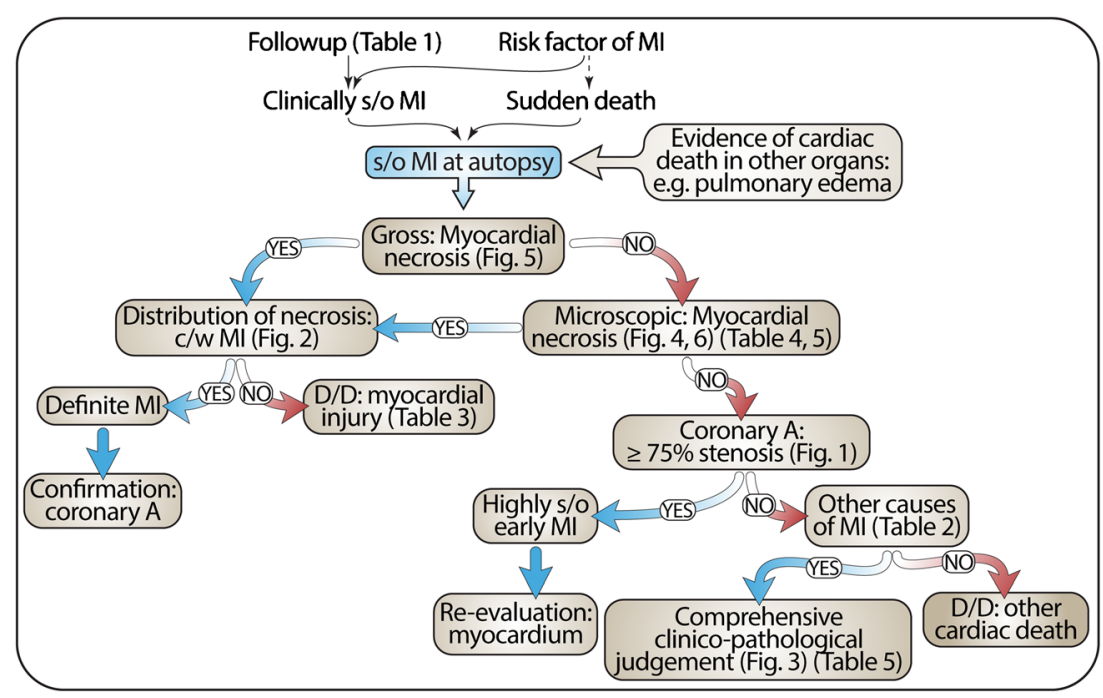

in the fibrous cap indicated by reduced immunopositivity of h-Caldesmon [9], the validity of which must be further verified in the future. In cases of sudden cardiac death, causes other than MI such as cardiomyopathy, channelopathy, and sarcoidosis must also be reconsidered $[2,3]$.

\section{Compliance with ethical standards}

Conflict of interest The author declares that he has no conflict of interest.

This is only partially applicable since the manuscript is an editorial and does not provide research data. Research involving human participants and/or animals is not reported. There was no need for an informed consent.

\section{References}

1. Michaud K, Basso C, d'Amati G et al (2019) Diagnosis of myocardial infarction at autopsy: AECVP reappraisal in the light of the current clinical classification. Virchow Arch. https://doi.org/10. 1007/s00428-019-02662-1

2. Green AC, Sheppard MN (2016) Sudden cardiac death associated with premature atheroma in the young: an autopsy study emphasising single-vessel lesions. Cardiol Young 26:743-748
3. Wang H, Yao Q, Zhu S, Zhang G, Wang Z, Li Z, Sun R, Lu C, Li C, $\mathrm{Pu} J$ (2014) The autopsy study of 553 cases of sudden cardiac death in Chinese adults. Heart Vessel 29:486-495

4. Sabatasso S, Mangin P, Fracasso T, Moretti M, Docquier M, Djonov V (2016) Early markers for myocardial ischemia and sudden cardiac death. Int J Legal Med 130:1265-1280

5. Leone O, Pieroni M, Rapezzi C, Olivotto I (2019) The spectrum of myocarditis: from pathology to the clinics. Virchows Arch 475:279301

6. Farb A, Tang AL, Burke AP, Sessums L, Liang Y, Virmani R (1995) Sudden coronary death. Frequency of active coronary lesions, inactive coronary lesions, and myocardial infarction. Circulation 92: 1701-1709

7. Takada A, Saito K, Ro A, Kobayashi M, Hamamatsu A, Murai T, Kuroda N (2003) Acute coronary syndrome as a cause of sudden death in patients with old myocardial infarction: a pathological analysis. Leg Med (Tokyo) 5 Suppl 1:S292-S294

8. Finn AV, Nakano M, Narula J, Kolodgie FD, Virmani R (2010) Concept of vulnerable/unstable plaque. Arterioscler Thromb Vasc Biol 30:1282-1292

9. Horita A, Kurata A, Ohno S, Shimoyamada H, Saito I, Kamma H, Kuroda M (2015) Immaturity of smooth muscle cells in the neointima is associated with acute coronary syndrome. Cardiovasc Pathol $24: 26-32$

Publisher's note Springer Nature remains neutral with regard to jurisdictional claims in published maps and institutional affiliations. 